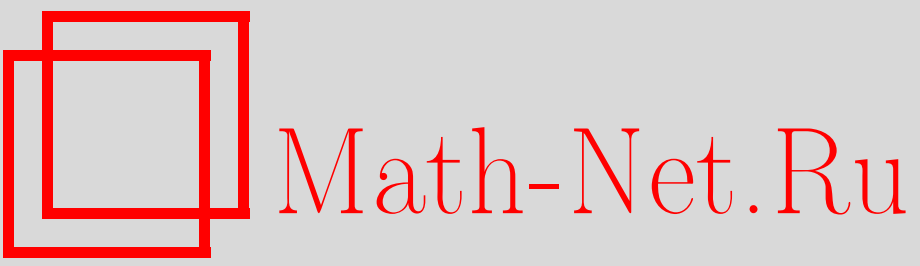

В. П. Спиридонов, Дерево Бэйли для интегралов, ТМФ, 2004, том 139, номер 1, 104-111

DOI: https://doi.org/10.4213/tmf45

Использование Общероссийского математического портала Math-Net.Ru подразумевает, что вы прочитали и согласны с пользовательским соглашением

http://www.mathnet.ru/rus/agreement

Параметры загрузки:

IP : 35.173 .219 .149

26 апреля 2023 г., 13:57:49 


\author{
ТЕОРЕТИЧЕСКАЯ \\ И МАТЕМАТИЧЕСКАЯ \\ ФИЗИКА \\ Том 139, № 1 \\ апрель, 2004
}

(C) 2004 г.

В.П. Спиридонов*

\title{
ДЕРЕВО БЭЙЛИ ДЛЯ ИНТЕГРАЛОВ
}

Вводится понятие интегральных пар Бэйли. С помощью одномерного эллиптического бета-интеграла построено бесконечное двоичное дерево тождеств для эллиптических гипергеометрических интегралов. Две специальные последовательности тождеств описаны в явном виде.

Ключевые слова: пары Бэйли, цепочки Бэйли, дерево Бэйли, бета-интегралы, эллиптические гипергеометрические интегралы.

Ряды и интегралы гипергеометрического типа имеют большое число приложений в математической физике. Поэтому изучение соответствующих специальных функций может быть важным для различных вычислений в теоретических моделях окружающей действительности. Цепочки Бэйли предоставляют мошные средства для порождения бесконечных последовательностей формул суммирования или преобразования для рядов гипергеометрического типа. Обзор соответствуюшего формализма и различные тождества для $q$-рядов, полученные с его помошью, приведены, например, в работах [1], [2]. Область применимости этой техники была недавно расширена на эллиптические гипергеометрические ряды [3]. Точнее, было найдено эллиптическое расширение вполне уравновешенной цепочки Бэйли, построенной Эндрюсом в [1], и были изучены ее простейшие следствия. Это позволило также обобшить одно из тождеств Эндрюса-Берковича [4] на эллиптический случай.

В процессе работы над статьей [3] автор пришел к принципиальному заключению, что должны существовать цепочки Бэйли для интегралов, но первые попытки построения простых примеров таких цепочек не увенчались успехом. Некоторое преобразование симметрии типа Бэйли для пары эллиптических гипергеометрических интегралов было предложено в работе [5]. Оказалось, что этот результат дает средства, необходимые для соответствуюшего обобшения формализма эллиптической цепочки Бэйли из работы [3]. В настоящей заметке сконструированы два примера цепочек Бэйли для интегралов с помощью наиболее сложного известного типа бета-интегралов одной переменной, а именно эллиптического бета-интеграла работы [6]. При совместном использовании эти цепочки образуют двоичное дерево тождеств для эллиптических гипергеометрических интегралов.

* Лаборатория теоретической физики им Н.Н. Боголюбова, Объединенный институт ядерных исследований, Дубна, Московская обл., Россия. E-mail: spiridon@thsun1.jinr.ru 
Обозначим через $\mathbb{T}$ положительно-ориентированную единичную окружность и возьмем две базовые переменные $q, p(|q|,|p|<1)$ и пять комплексных параметров $t_{m}, m=$ $0, \ldots, 4$, удовлетворяющих неравенствам $\left|t_{m}\right|<1,|p q|<|A|$, где $A \equiv \prod_{r=0}^{4} t_{r}$. Тогда эллиптический бета-интеграл

$$
\mathcal{N}_{E}(\mathbf{t})=\frac{1}{2 \pi i} \int_{\mathbb{T}} \frac{\prod_{m=0}^{4} \Gamma\left(z^{ \pm} t_{m}\right)}{\Gamma\left(z^{ \pm 2}, z^{ \pm} A\right)} \frac{d z}{z}
$$

допускает точное вычисление [6]:

$$
\mathcal{N}_{E}(\mathbf{t})=\frac{2 \prod_{0 \leqslant m<s \leqslant 4} \Gamma\left(t_{m} t_{s}\right)}{(q ; q)_{\infty}(p ; p)_{\infty} \prod_{m=0}^{4} \Gamma\left(A t_{m}^{-1}\right)}
$$

Мы используем стандартное обозначение $(q ; q)_{\infty}=\prod_{n=1}^{\infty}\left(1-q^{n}\right)$ и следуюшие условные соглашения:

$$
\begin{aligned}
\Gamma\left(t z^{ \pm}\right) & =\Gamma\left(t z, t z^{-1}\right), \quad \Gamma\left(z^{ \pm 2}\right)=\Gamma\left(z^{2}, z^{-2}\right) \\
\Gamma\left(t z^{ \pm} x^{ \pm}\right) & =\Gamma\left(t z x, t z x^{-1}, t z^{-1} x, t z^{-1} x^{-1}\right) \\
\Gamma\left(t_{1}, \ldots, t_{m}\right) & \equiv \prod_{r=1}^{m} \Gamma\left(t_{r} ; q, p\right)
\end{aligned}
$$

где $\Gamma(z ; q, p)$ обозначает эллиптическую гамма-функцию

$$
\Gamma(z ; q, p)=\prod_{j, k=0}^{\infty} \frac{1-z^{-1} q^{j+1} p^{k+1}}{1-z q^{j} p^{k}}
$$

(краткое историческое описание этой функции и соответствующие литературные ссылки приведены, например, в [5]). При $p=0$ формула (1) сводится к $q$-бета-интегралу Нассраллаха-Рахмана, который может быть упрощен до интеграла Аски-Вильсона [7].

ОПРЕДЕЛЕНИЕ. Две функции $\alpha(z, t)$ и $\beta(z, t), z, t \in \mathbb{C}$, образуют интегральную әллиптическую пару Бэйли по отношению к параметру $t$, если они удовлетворяют следуюшему равенству:

$$
\beta(w, t)=\kappa \int_{\mathbb{T}} \Gamma\left(t w^{ \pm} z^{ \pm}\right) \alpha(z, t) \frac{d z}{z}, \quad \kappa \equiv \frac{(p ; p)_{\infty}(q ; q)_{\infty}}{4 \pi i}
$$

Предположим, что мы нашли частный пример интегральной эллиптической пары Бэйли. Тогда сушествует способ построения бесконечной последовательности таких пар исходя из заданной пары. 
ТЕОрема 1. Для всякой пары функиий $\alpha(z, t)$ и $\beta(z, t)$, образующей интегральную әллиптическую пару Бэйли по отношению $\kappa t$, новые функции, определенные для $w \in \mathbb{T} \kappa a \kappa$

$$
\alpha^{\prime}(w, s t)=\frac{\Gamma\left(t u w^{ \pm}\right)}{\Gamma\left(t s^{2} u w^{ \pm}\right)} \alpha(w, t)
$$

$$
\beta^{\prime}(w, s t)=\kappa \frac{\Gamma\left(t^{2} s^{2}, t^{2} s u w^{ \pm}\right)}{\Gamma\left(s^{2}, t^{2}, s u w^{ \pm}\right)} \int_{\mathbb{T}} \frac{\Gamma\left(s w^{ \pm} x^{ \pm}, u x^{ \pm}\right)}{\Gamma\left(x^{ \pm 2}, t^{2} s^{2} u x^{ \pm}\right)} \beta(x, t) \frac{d x}{x},
$$

образуют интегральную әллиптическую пару Бәйли по отношению $к$ параметру st. Здесь $t, s, u$ являются произвольными комплексными параметрами, удовлетворяющими ограничениям $|t|,|s|,|u|<1,|p q|<\left|t^{2} s^{2} u\right|$.

ДокАЗАТЕЛЬСтво. Подставим определение (4) в соотношение (6):

$$
\beta^{\prime}(w, s t)=\kappa^{2} \frac{\Gamma\left(t^{2} s^{2}, t^{2} s u w^{ \pm}\right)}{\Gamma\left(s^{2}, t^{2}, s u w^{ \pm}\right)} \int_{\mathbb{T}^{2}} \frac{\Gamma\left(s w^{ \pm} x^{ \pm}, t z^{ \pm} x^{ \pm}, u x^{ \pm}\right)}{\Gamma\left(x^{ \pm 2}, t^{2} s^{2} u x^{ \pm}\right)} \alpha(z, t) \frac{d z}{z} \frac{d x}{x} .
$$

Поскольку интегрируемая функция ограничена на $\mathbb{T}$, мы можем изменить порядок интегрирований (т.е. проинтегрировать сначала по $x$ ) и применить формулу (1). Это дает

$$
\begin{aligned}
\beta^{\prime}(w, s t) & =\kappa \int_{\mathbb{T}} \frac{\Gamma\left(s t w^{ \pm} z^{ \pm}, t u z^{ \pm}\right)}{\Gamma\left(t s^{2} u z^{ \pm}\right)} \alpha(z, t) \frac{d z}{z}= \\
& =\kappa \int_{\mathbb{T}} \Gamma\left(s t w^{ \pm} z^{ \pm}\right) \alpha^{\prime}(z, s t) \frac{d z}{z}
\end{aligned}
$$

т.е. функции $\alpha^{\prime}(w, s t)$ и $\beta^{\prime}(w, s t)$ образуют интегральную пару Бэйли по отношению к параметру $s t$

В некотором смысле эта теорема может рассматриваться как интегральная лемма Бэйли.

Подставив в интеграл (1) соотношения $t_{3}=t w, t_{4}=t w^{-1}$, нетрудно найти начальную интегральную эллиптическую пару Бэйли:

$$
\begin{aligned}
\alpha(z, t) & =\frac{\prod_{r=0}^{2} \Gamma\left(t_{r} z^{ \pm}\right)}{\Gamma\left(z^{ \pm 2}, t^{2} t_{0} t_{1} t_{2} z^{ \pm}\right)}, \\
\beta(w, t) & =\Gamma\left(t^{2}\right) \prod_{0 \leqslant r<j \leqslant 2} \frac{\Gamma\left(t_{r} t_{j}\right)}{\Gamma\left(t^{2} t_{r} t_{j}\right)} \frac{\prod_{r=0}^{2} \Gamma\left(t t_{r} w^{ \pm}\right)}{\Gamma\left(t t_{0} t_{1} t_{2} w^{ \pm}\right)},
\end{aligned}
$$

где $|t|,\left|t_{r}\right|<1,|p q|<\left|t^{2} t_{0} t_{1} t_{2}\right|$. Подставляя эти выражения в цепочечные правила (5) и (6), мы получаем

$$
\begin{aligned}
\alpha^{\prime}(z, s t)= & \frac{\Gamma\left(t u z^{ \pm}\right) \prod_{r=0}^{2} \Gamma\left(t_{r} z^{ \pm}\right)}{\Gamma\left(t s^{2} u z^{ \pm}, z^{ \pm 2}, t^{2} t_{0} t_{1} t_{2} z^{ \pm}\right)}, \\
\beta^{\prime}(w, s t)= & \kappa \frac{\Gamma\left(t^{2} s^{2}, t^{2} s u w^{ \pm}\right)}{\Gamma\left(s^{2}, s u w^{ \pm}\right)} \prod_{0 \leqslant r<j \leqslant 2} \frac{\Gamma\left(t_{r} t_{j}\right)}{\Gamma\left(t^{2} t_{r} t_{j}\right)} \times \\
& \times \int_{\mathbb{T}} \frac{\Gamma\left(s w^{ \pm} x^{ \pm}, u x^{ \pm}\right) \prod_{r=0}^{2} \Gamma\left(t t_{r} x^{ \pm}\right)}{\Gamma\left(x^{ \pm 2}, t^{2} s^{2} u x^{ \pm}, t t_{0} t_{1} t_{2} x^{ \pm}\right)} \frac{d x}{x} .
\end{aligned}
$$


Будучи подставленными в основное генерирующее соотношение (8), эти функции порождают преобразование симметрии для двух эллиптических гипергеометрических интегралов:

$$
\begin{aligned}
& \prod_{j=0}^{2} \frac{\Gamma\left(B t_{j}^{-1}\right)}{\Gamma\left(t^{2} B t_{j}^{-1}\right)} \int_{\mathbb{T}} \frac{\prod_{j=1}^{3} \Gamma\left(t t_{j} z^{ \pm}, s_{j} z^{ \pm}\right)}{\Gamma\left(z^{ \pm 2}, t^{2} S z^{ \pm}, t B z^{ \pm}\right)} \frac{d z}{z}= \\
& \quad=\prod_{j=0}^{2} \frac{\Gamma\left(S s_{j}^{-1}\right)}{\Gamma\left(t^{2} S s_{j}^{-1}\right)} \int_{\mathbb{T}} \frac{\prod_{j=1}^{3} \Gamma\left(t s_{j} z^{ \pm}, t_{j} z^{ \pm}\right)}{\Gamma\left(z^{ \pm 2}, t^{2} B z^{ \pm}, t S z^{ \pm}\right)} \frac{d z}{z}
\end{aligned}
$$

где $s_{0}=u, s_{1}=s w, s_{2}=s w^{-1}$ и $B=\prod_{j=0}^{2} t_{j}, S=\prod_{j=0}^{2} s_{j}$. Тождество (10) было установлено в работе [5], и интегральная эллиптическая цепочка Бэйли (4)-(6) построена путем обобщения соответствующего способа вывода этого равенства.

Итеративное применение правил (5) и (6) порождает бесконечную цепочку тождеств для эллиптических гипергеометрических интегралов. Результат $m$-кратных итераций имеет вид

$$
\begin{aligned}
& \alpha^{(m)}\left(x, \prod_{l=1}^{m} s_{l} t\right)=\prod_{k=1}^{m} \frac{\Gamma\left(t \prod_{l=1}^{k-1} s_{l} u_{k} x^{ \pm}\right)}{\Gamma\left(t \prod_{l=1}^{k-1} s_{l} s_{k}^{2} u_{k} x^{ \pm}\right)} \alpha^{(0)}(x, t), \\
& \beta^{(m)}\left(x_{m+1}, \prod_{l=1}^{m} s_{l} t\right)=\kappa^{m} \prod_{k=1}^{m} \frac{\Gamma\left(t^{2} \prod_{l=1}^{k} s_{l}^{2}\right)}{\Gamma\left(s_{k}^{2}, t^{2} \prod_{l=1}^{k-1} s_{l}^{2}\right)} \times \\
& \quad \times \int_{\mathbb{T}^{m}} \prod_{k=1}^{m} \frac{\Gamma\left(t^{2} \prod_{l=1}^{k-1} s_{l}^{2} s_{k} u_{k} x_{k+1}^{ \pm}, s_{k} x_{k+1}^{ \pm} x_{k}^{ \pm}, u_{k} x_{k}^{ \pm}\right)}{\Gamma\left(s_{k} u_{k} x_{k+1}^{ \pm}, x_{k}^{ \pm 2}, t^{2} \prod_{l=1}^{k} s_{l}^{2} u_{k} x_{k}^{ \pm}\right)} \beta^{(0)}\left(x_{1}, t\right) \prod_{l=1}^{m} \frac{d x_{l}}{x_{l}} .
\end{aligned}
$$

Используя выражения $(9)$ в качестве начальных функций $\alpha^{(0)}(x, t)$ и $\beta^{(0)}(x, t)$ и подставляя (11) в (4), мы получаем тождество

$$
\begin{aligned}
\kappa^{m-1} & \prod_{k=1}^{m} \frac{\Gamma\left(t^{2} \prod_{l=1}^{k} s_{l}^{2}\right)}{\Gamma\left(s_{k}^{2}, t^{2} \prod_{l=1}^{k-1} s_{l}^{2}\right)} \int_{\mathbb{T}^{m}} \frac{\prod_{r=0}^{2} \Gamma\left(t t_{r} x_{1}^{ \pm}\right)}{\Gamma\left(t t_{0} t_{1} t_{2} x_{1}^{ \pm}\right)} \times \\
& \times \prod_{k=1}^{m} \frac{\Gamma\left(t^{2} \prod_{l=1}^{k-1} s_{l}^{2} s_{k} u_{k} x_{k+1}^{ \pm}, s_{k} x_{k+1}^{ \pm} x_{k}^{ \pm}, u_{k} x_{k}^{ \pm}\right)}{\Gamma\left(s_{k} u_{k} x_{k+1}^{ \pm}, x_{k}^{ \pm 2}, t^{2} \prod_{l=1}^{k} s_{l}^{2} u_{k} x_{k}^{ \pm}\right)} \frac{d x_{l}}{x_{l}}= \\
= & \frac{1}{\Gamma\left(t^{2}\right)} \prod_{0 \leqslant r<j \leqslant 2} \frac{\Gamma\left(t^{2} t_{r} t_{j}\right)}{\Gamma\left(t_{r} t_{j}\right)} \int_{\mathbb{T}} \frac{\Gamma\left(t \prod_{l=1}^{m} s_{l} x_{m+1}^{ \pm} x^{ \pm}\right) \prod_{r=0}^{2} \Gamma\left(t_{r} x^{ \pm}\right)}{\Gamma\left(x^{ \pm 2}, t^{2} t_{0} t_{1} t_{2} x^{ \pm}\right)} \times \\
& \times \prod_{k=1}^{m} \frac{\Gamma\left(t \prod_{l=1}^{k-1} s_{l} u_{k} x^{ \pm}\right)}{\Gamma\left(t \prod_{l=1}^{k-1} s_{l} s_{k}^{2} u_{k} x^{ \pm}\right)} \frac{d x}{x},
\end{aligned}
$$

которое для $m=1$ сводится к (10). В некотором смысле соотношение (12) представляет собой обобшение высокого уровня тождеств типа Роджерса-Рамануджана для многократных рядов, которые были выведены в работе [8]. Интегральные аналоги некоторых 
из тождеств Роджерса-Рамануджана были рассмотрены в работе [9]. Учитывая многочисленность исследований, касающихся цепочек Бэйли для рядов, тот факт, что их интегральные аналоги не получили соответствуюшего внимания в литературе, представляется удивительным. Некоторые примеры однократных преобразований типа Бэйли для интегралов были известны в течение долгого времени. Фактически все изменения порядков интегрирований в многократных интегралах, использованных в [10], [5], могут быть интерпретированы как интегральные обобщения изменений порядка суммирований для рядов [1], [2]. Обшая идея использования изменения порядка интегрирований для получения полезной информации об интегралах восходит к методам вывода обычного бета-интеграла Пуассона и Якоби [11].

Известно, что для определенных пар Бэйли существует несколько различных цепочечных правил подстановки (или лемм Бэйли). В этом случае совокупность получаюшихся тождеств имеет более сложную структуру, которая называется решеткой [12] или деревом Бэйли [1]. Оказывается, что в нашем случае можно расширить интегральную цепочку Бэйли, описанную в теореме 1 , до структуры дерева благодаря дополнительной интегральной лемме Бэйли, описываемой ниже.

ТЕОРема 2. Для любых двух функций $\alpha(z, t)$ и $\beta(z, t)$, образующих интегральную әллиптическую пару Бәйли по отношению $\kappa t,|t|<1$, новъе функции, определенньве для $w \in \mathbb{T}$ как

$$
\alpha^{\prime}(w, t)=\kappa \frac{\Gamma\left(s^{2} t^{2}, u w^{ \pm}\right)}{\Gamma\left(s^{2}, t^{2}, w^{ \pm 2}, t^{2} s^{2} u w^{ \pm}\right)} \int_{\mathbb{T}} \frac{\Gamma\left(t^{2} s u x^{ \pm}, s w^{ \pm} x^{ \pm}\right)}{\Gamma\left(s u x^{ \pm}\right)} \alpha(x, s t) \frac{d x}{x}
$$

$u$

$$
\beta^{\prime}(w, t)=\frac{\Gamma\left(t u w^{ \pm}\right)}{\Gamma\left(t s^{2} u w^{ \pm}\right)} \beta(w, s t),
$$

также образуют интегральную әллиптическую пару Бәйли по отношению $\kappa t$. Здесь $s$, и являютося произвольными комплексными параметрами (они не связаны c s, u, фигурирующими в теореме 1), удовлетворяюшими ограничениям $|s|,|u|<1$, $|p q|<\left|t^{2} s^{2} u\right|$.

ДокАЗАТЕльСтво. Проверка справедливости этого утверждения подобна доказательству теоремы 1 . Для этого необходимо заменить $\alpha(w, t)$ и $\beta(w, t)$ в равенстве $(4)$ на $\alpha^{\prime}(w, t)$ и $\beta^{\prime}(w, t)$, подставить в него соотношение (13), изменить порядок интегрирований и применить формулу (2). Мы опускаем детали рассмотрения ввиду их простоты.

В работе [3] была найдена только одна лемма Бэйли для эллиптических гипергеометрических рядов. Теорема 1 была получена по некоторой эвристической аналогии с ней. Естественно ожидать, что существует некоторый предел, который позволил бы перейти от теоремы 1 к соответствуюшей цепочке Бэйли для рядов. Очевидно, что теорема 2 также должна иметь некоторый аналог для рядов, который привел бы к эллиптическому дереву Бэйли для рядов (его аналог для $q$-гипергеометрических рядов качественно отличался бы от дерева, рассмотренного в работах [1], [4]).

В качестве иллюстрации мы опишем одну частную последовательность тождеств, порожденных сконструированным деревом Бэйли. Для этого возьмем функции (9) и 
применим к ним преобразования (13) и (14) с параметрами $s=s_{1}, u=u_{1}$. K результирующим функциям $\alpha^{\prime}(w, t)$ и $\beta^{\prime}(w, t)$ мы применяем преобразования (5) и (6) с параметрами $s=s_{2}, u=u_{2}$. В итоге получаем

$$
\begin{aligned}
\alpha^{\prime \prime}\left(w, s_{2} t\right)= & \kappa \frac{\Gamma\left(t^{2} s_{1}^{2}, t u_{2} w^{ \pm}, u_{1} w^{ \pm}\right)}{\Gamma\left(s_{1}^{2}, t^{2}, w^{ \pm 2}, t s_{2}^{2} u_{2} w^{ \pm}, t^{2} s_{1}^{2} u_{1} w^{ \pm}\right)} \times \\
& \times \int_{\mathbb{T}} \frac{\Gamma\left(t^{2} s_{1} u_{1} x^{ \pm}, s_{1} w^{ \pm} x^{ \pm}\right) \prod_{r=0}^{2} \Gamma\left(t_{r} x^{ \pm}\right)}{\Gamma\left(x^{ \pm 2}, s_{1} u_{1} x^{ \pm}, t^{2} s_{1}^{2} t_{0} t_{1} t_{2} x^{ \pm}\right)} \frac{d x}{x}, \\
\beta^{\prime \prime}\left(w, s_{2} t\right)= & \kappa \frac{\Gamma\left(t^{2} s_{2}^{2}, t^{2} s_{1}^{2}, t^{2} s_{2} u_{2} w^{ \pm}\right)}{\Gamma\left(s_{2}^{2}, t^{2}, s_{2} u_{2} w^{ \pm}\right)} \prod_{0 \leqslant r<j \leqslant 2} \frac{\Gamma\left(t_{r} t_{j}\right)}{\Gamma\left(t^{2} s_{1}^{2} t_{r} t_{j}\right)} \times \\
& \times \int_{\mathbb{T}} \frac{\Gamma\left(s_{2} w^{ \pm} x^{ \pm}, t u_{1} x^{ \pm}, u_{2} x^{ \pm}\right) \prod_{r=0}^{2} \Gamma\left(t s_{1} t_{r} x^{ \pm}\right)}{\Gamma\left(x^{ \pm 2}, t^{2} s_{2}^{2} u_{2} x^{ \pm}, t s_{1}^{2} u_{1} x^{ \pm}, t s_{1} t_{0} t_{1} t_{2} x^{ \pm}\right)} \frac{d x}{x} .
\end{aligned}
$$

Подставляя эти функции в основное соотношение (4) с параметром $t$, замененным на $s_{2} t$, мы получаем равенство для двух интегралов

$$
\begin{gathered}
\int_{\mathbb{T}} \frac{\Gamma\left(s_{2} w^{ \pm} x^{ \pm}, t u_{1} x^{ \pm}, u_{2} x^{ \pm}\right) \prod_{r=0}^{2} \Gamma\left(t s_{1} t_{r} x^{ \pm}\right)}{\Gamma\left(x^{ \pm 2}, t^{2} s_{2}^{2} u_{2} x^{ \pm}, t s_{1}^{2} u_{1} x^{ \pm}, t s_{1} t_{0} t_{1} t_{2} x^{ \pm}\right)} \frac{d x}{x}= \\
=\kappa \prod_{0 \leqslant r<j \leqslant 2} \frac{\Gamma\left(t^{2} s_{1}^{2} t_{r} t_{j}\right)}{\Gamma\left(t_{r} t_{j}\right)} \frac{\Gamma\left(s_{2}^{2}, s_{2} u_{2} w^{ \pm}\right)}{\Gamma\left(s_{1}^{2}, t^{2} s_{2}^{2}, t^{2} s_{2} u_{2} w^{ \pm}\right)} \times \\
\quad \times \int_{\mathbb{T}^{2}} \Gamma\left(s_{1} x_{2}^{ \pm} x_{1}^{ \pm}\right) \frac{\Gamma\left(t s_{2} w^{ \pm} x_{2}^{ \pm}, t u_{2} x_{2}^{ \pm}, u_{1} x_{2}^{ \pm}\right)}{\Gamma\left(x_{2}^{ \pm 2}, t s_{2}^{2} u_{2} x_{2}^{ \pm}, t^{2} s_{1}^{2} u_{1} x_{2}^{ \pm}\right)} \times \\
\quad \times \frac{\Gamma\left(t^{2} s_{1} u_{1} x_{1}^{ \pm}\right) \prod_{r=0}^{2} \Gamma\left(t_{r} x_{1}^{ \pm}\right)}{\Gamma\left(x_{1}^{ \pm 2}, s_{1} u_{1} x_{1}^{ \pm}, t^{2} s_{1}^{2} t_{0} t_{1} t_{2} x_{1}^{ \pm}\right)} \frac{d x_{1}}{x_{1}} \frac{d x_{2}}{x_{2}} .
\end{gathered}
$$

Это тождество было получено при условии, что все параметры лежат внутри единичной окружности, и при $\left|t^{2} s_{1}^{2} t_{0} t_{1} t_{2}\right|,\left|t^{2} s_{1}^{2} u_{2}\right|,\left|t^{2} s_{2}^{2} u_{2}\right|>|p q|$. Однако благодаря аналитичности оно остается справедливым для более широкой области значений параметров при условии, что мы заменим окружность $\mathbb{T}$ контурами интегрирования, которые охватывают последовательности полюсов интегрируемой функции, сходяшиеся к нулю, и исключают другие полюсы.

Нетрудно найти результат $m$-кратной итерации преобразований (13) и (14) с различными параметрами $u_{k}, s_{k}$ :

$$
\begin{aligned}
\alpha^{(m)}\left(x_{m+1}, t\right)= & \kappa^{m} \prod_{k=1}^{m} \frac{\Gamma\left(\prod_{l=k}^{m} s_{l}^{2} t^{2}\right)}{\Gamma\left(s_{k}^{2}, \prod_{l=k+1}^{m} s_{l}^{2} t^{2}\right)} \times \\
& \times \int_{\mathbb{T}^{m}} \prod_{k=1}^{m} \frac{\Gamma\left(u_{k} x_{k+1}^{ \pm}, \prod_{l=k+1}^{m} s_{l}^{2} t^{2} s_{k} u_{k} x_{k}^{ \pm}, s_{k} x_{k+1}^{ \pm} x_{k}^{ \pm}\right)}{\Gamma\left(x_{k+1}^{ \pm 2}, \prod_{l=k}^{m} s_{l}^{2} t^{2} u_{k} x_{k+1}^{ \pm}, s_{k} u_{k} x_{k}^{ \pm}\right)} \times \\
& \times \alpha^{(0)}\left(x_{1}, \prod_{l=1}^{m} s_{l} t\right) \frac{d x_{1}}{x_{1}} \cdots \frac{d x_{m}}{x_{m}}, \\
\beta^{(m)}(w, t)= & \prod_{k=1}^{m} \frac{\Gamma\left(t \prod_{l=k+1}^{m} s_{l} u_{k} w^{ \pm}\right)}{\Gamma\left(t \prod_{l=k+1}^{m} s_{l} s_{k}^{2} u_{k} w^{ \pm}\right)} \beta^{(0)}\left(w, \prod_{l=1}^{m} s_{l} t\right) .
\end{aligned}
$$


Применение к этим функциям преобразований (5) и (6) с параметрами $s=s_{m+1}, u=$ $u_{m+1}$ дает

$$
\begin{aligned}
\alpha^{\prime}\left(x_{m+1}, s_{m+1} t\right)= & \frac{\Gamma\left(t u_{m+1} x_{m+1}^{ \pm}\right)}{\Gamma\left(t s_{m+1}^{2} u_{m+1} x_{m+1}^{ \pm}\right)} \alpha^{(m)}\left(x_{m+1}, t\right) \\
\beta^{\prime}\left(w, s_{m+1} t\right)= & \kappa \frac{\Gamma\left(t^{2} s_{m+1}^{2}, t^{2} s_{m+1} u_{m+1} w^{ \pm}\right)}{\Gamma\left(s_{m+1}^{2}, t^{2}, s_{m+1} u_{m+1} w^{ \pm}\right)} \times \\
& \times \int_{\mathbb{T}} \frac{\Gamma\left(s_{m+1} w^{ \pm} x^{ \pm}, u_{m+1} x^{ \pm}\right)}{\Gamma\left(x^{ \pm 2}, t^{2} s_{m+1}^{2} u_{m+1} x^{ \pm}\right)} \beta^{(m)}(x, t) \frac{d x}{x}= \\
= & \kappa \int_{\mathbb{T}} \Gamma\left(s_{m+1} t w^{ \pm} x_{m+1}^{ \pm}\right) \alpha^{\prime}\left(x_{m+1}, s_{m+1} t\right) \frac{d x_{m+1}}{x_{m+1}} .
\end{aligned}
$$

Мы подставляем в последнее соотношение выражения (16) с функциями (9), выступающими в качестве $\alpha^{(0)}(w, t)$ и $\beta^{(0)}(w, t)$. В результате получаем тождество

$$
\begin{aligned}
\int_{\mathbb{T}} \frac{\Gamma\left(s_{m+1} w^{ \pm} x^{ \pm}, u_{m+1} x^{ \pm}\right)}{\Gamma\left(x^{ \pm 2}, t^{2} s_{m+1}^{2} u_{m+1} x^{ \pm}\right)} \prod_{k=1}^{m} \frac{\Gamma\left(t \prod_{l=k+1}^{m} s_{l} u_{k} x^{ \pm}\right)}{\Gamma\left(t \prod_{l=k+1}^{m} s_{l} s_{k}^{2} u_{k} x^{ \pm}\right)} \frac{\prod_{r=0}^{2} \Gamma\left(t \prod_{k=1}^{m} s_{k} t_{r} x^{ \pm}\right)}{\Gamma\left(t \prod_{k=1}^{m} s_{k} t_{0} t_{1} t_{2} x^{ \pm}\right)} \frac{d x}{x}= \\
=\frac{\kappa^{m} \Gamma\left(s_{m+1}^{2}, t^{2}, s_{m+1} u_{m+1} w^{ \pm}\right)}{\Gamma\left(t^{2} s_{m+1}^{2}, t^{2} \prod_{k=1}^{m} s_{k}^{2}, t^{2} s_{m+1} u_{m+1} w^{ \pm}\right)} \times \\
\quad \times \prod_{k=1}^{m} \frac{\Gamma\left(\prod_{l=k}^{m} s_{l}^{2} t^{2}\right)}{\Gamma\left(s_{k}^{2}, \prod_{l=k+1}^{m} s_{l}^{2} t^{2}\right)} \prod_{0 \leqslant r<j \leqslant 2} \frac{\Gamma\left(t^{2} \prod_{k=1}^{m} s_{k}^{2} t_{r} t_{j}\right)}{\Gamma\left(t_{r} t_{j}\right)} \times \\
\quad \times \int_{\mathbb{T}^{m+1}} \frac{\Gamma\left(t s_{m+1} w^{ \pm} x_{m+1}^{ \pm}, t u_{m+1} x_{m+1}^{ \pm}\right) \prod_{r=0}^{2} \Gamma\left(t_{r} x_{1}^{ \pm}\right)}{\Gamma\left(t s_{m+1}^{2} u_{m+1} x_{m+1}^{ \pm}, x_{1}^{ \pm 2}, t^{2} \prod_{k=1}^{m} s_{k}^{2} t_{0} t_{1} t_{2} x_{1}^{ \pm}\right)} \times \\
\quad \times \prod_{k=1}^{m} \frac{\Gamma\left(u_{k} x_{k+1}^{ \pm}, \prod_{l=k+1}^{m} s_{l}^{2} t^{2} s_{k} u_{k} x_{k}^{ \pm}, s_{k} x_{k+1}^{ \pm} x_{k}^{ \pm}\right)}{\Gamma\left(x_{k+1}^{ \pm 2}, \prod_{l=k}^{m} s_{l}^{2} t^{2} u_{k} x_{k+1}^{ \pm}, s_{k} u_{k} x_{k}^{ \pm}\right)} \frac{d x_{1}}{x_{1}} \ldots \frac{d x_{m+1}}{x_{m+1}} .
\end{aligned}
$$

Для $m=1$ это равенство сводится к соотношению (15).

Очевидно, что необходимо провести более тщательный анализ следствий полученного эллиптического дерева Бэйли для интегралов с различными кратностями интегрирования. В частности, единственность этого дерева является интересной проблемой. Следствия для $q$-гипергеометрических интегралов и для эллиптических и $q$-гипергеометрических рядов также заслуживают подробного изучения.

Благодарности. Данная работа частично поддержана Российским фондом фундаментальных исследований (грант № 03-01-00780). 


\section{Список литературы}

[1] G. E. Andrews. Bailey's transform, lemma, chains and tree. In: Proc. NATO ASI Special functions-2000 (Tempe, USA, May 29-June 9, 2000). Eds. J. Bustoz, M. E. H. Ismail, S. K. Suslov. Dordrecht: Kluwer, 2001. P. 1-22.

[2] S. O. Warnaar. 50 years of Bailey's lemma. In: Algebraic Combinatorics and Applications. Ed. A. Betten. Berlin: Springer, 2001. P. 333-347.

[3] V.P. Spiridonov. Int. Math. Res. Notices. 2002. V. 37. P. 1945-1977.

[4] G. E. Andrews, A. Berkovich. J. London Math. Soc. (2). 2002. V. 66. P. 529-549.

[5] V.P. Spiridonov. Theta hypergeometric integrals Алгебра и анализ. 2003. V. 15. № 6 (в печати).

[6] V.P. Spiridonov. An elliptic beta integral. In: Proc. Fifth Internat. Conf. on Difference Equations (Temuco, Chile, January 3-7, 2000). Eds. S. Elaydi, J. Lopez Fenner, G. Ladas, M. Pinto. London: Taylor and Francis, 2001. Р. 273-282; В. П. Спиридонов. УМН. 2001. Т. 56. № 1. C. $181-182$.

[7] G. Gasper, M. Rahman. Basic Hypergeometric Series. Encyclopedia of Mathematics and its Applications. V. 35. Cambridge: Cambridge Univ. Press, 1990.

[8] G. E. Andrews. Pacific J. Math. 1984. V. 114. P. 267-283.

[9] K. Garrett, M. E. H. Ismail, D. Stanton. Adv. Appl. Math. 1999. V. 23. P. 274-299.

[10] G. Anderson. Forum Math. 1991. V. 3. P. 415-417; R. Y. Denis, R. A. Gustafson. SIAM J. Math. Anal. 1992. V. 23. P. 552-561; R. A. Gustafson. SIAM J. Math. Anal. 1994. V. 25. P. 441-449; R. A. Gustafson, M. A. Rakha. Ann. Comb. 2000. V. 4. P. 347-373; J.F. van Diejen, V. P. Spiridonov. Int. Math. Res. Notices. 2001. V. 20. P. 1083-1110.

[11] G. E. Andrews, R. Askey, R. Roy. Special Functions. Encyclopedia of Mathematics and its Applications. V. 71. Cambridge: Cambridge Univ. Press, 1999.

[12] A. K. Agarwal, G. E. Andrews, D. M. Bressoud. J. Indian Math. Soc. 1987. V. 51. P. 57-73. 